\title{
A new compact hydraulic propeller turbine for low heads
}

\author{
Dario Barsi ${ }^{1}$, Marina Ubaldi $^{1}$, Pietro Zunino ${ }^{1, *}$, and Robert Fink ${ }^{2}$ \\ ${ }^{1}$ University of Genova, Department of Mechanical, Energy, Management and Transportation \\ Engineering, Genova, Italy \\ ${ }^{2}$ SEMI Industrial Ltda, Saõ Paulo, Brazil
}

\begin{abstract}
In this paper, a design procedure for a compact hydraulic propeller turbine for low heads is proposed and developed. The design is based upon classical criteria and on the employment of statistical correlations, which relates the main geometrical parameters to the fundamental design parameters of turbomachinery. The procedure for obtaining the meridional flow path and the stator vanes and rotor blades geometries is explained step by step. The obtained 3D model is employed to carry out a CFD calculation in order to obtain the turbine overall performance and main working parameters.
\end{abstract}

\section{Introduction}

Hydraulic energy conversion using gravitational field is one of the most traditional and low polluting way of energy conversion, which still accounts for $16.5 \%$ of the world global electric energy conversion balance [1]. Hydraulic energy remains also the most diffused, in terms of TWh produced, among the renewable energy sources, due to the good availability of the conversion fluid employed, the water, and the highest specific energy among the renewables, related to the net head available, $H_{n}$. Nowadays, many of the most easily and economically exploitable projects have already been developed and, therefore, some less economically convenient sites as those with very low net heads (in the range $2 \mathrm{~m}<H_{n}<8 \mathrm{~m}$, approximately) have started to be taken in consideration. The main technical and economic problems are related to the low specific energy associated to the low head, which in turns results into the necessity of larger flow rate, $Q$, for achieving a reasonable amount of power, and, at the same time, low water velocity, which is proportional to the net head square root. Both conditions bring to a larger diameter of the turbine, which means larger costs and lower rotational speeds. For this reason, recently, new, very compact, simplified, modular, easy to manufacture and cost reduced hydraulic turbine conceptual prototypes have been developed by the main hydraulic turbine manufacturers. See for instance the turbine series StreamDiver ${ }^{\circledR}$ from Voith [2] and the turbine series Hydromatrix ${ }^{\circledR}$ from Andritz Hydro [3].

\footnotetext{
*Corresponding author: pietro.zunino@unige.it
} 
All these turbine modular series are characterized by high specific speed axial propeller rotors with rotor fixed blades and a fixed distribution, which determine, for a fixed rotational speed, a unique operating point. Therefore, the design objective, for a given turbine diameter, is to obtain the largest as possible flow rate compatible with an acceptable hydraulic efficiency. All these turbine series are characterized by a very compact and simplified (where possible) hydraulic design, very compact but robust and accurate mechanics, and, furthermore, they are modular and easy to manufacture and to install. The turbine rotor is directly coupled with a permanent magnet, synchronous electric generator enclosed in a waterproof, cylindrical front-end bulb located at the turbine intake. The rotational speed is set in relation to the net head (which determines the water velocities within the turbine) and can be varied if the head will change during the operation. An electronic converter provides the electric current at the correct grid frequency. Recently, SEMI Industrial, a mid-size Brazilian industrial company, producer of advanced hydraulic turbine systems, is being developing a new compact modular axial hydraulic turbine for low heads, named Hydrocell ${ }^{\circledR}$, in cooperation with the University of Genova. The present paper reports and explains the hydraulic design procedure which is based on 1D and 2D well established procedures, design criteria and correlations and 3D CFD advanced simulations in order to obtain the targeted operating conditions and maximize the efficiency as reported in similar works such as [4-6].

\section{Design criteria}

The concept of specific speed, $n_{q}$, is often utilized for classification and design of turbomachinery. For hydraulic turbines and pumps, it is always used as the main reference parameter. From the physical point of view, work and therefore aerodynamic loading of blades and, as a consequence, hydraulic losses, mechanical stress, cavitation characteristics are all related to the net head. The Thoma cavitation coefficient [7], denoted with $\sigma$, is directly related to the specific speed and increases more than linearly with $n_{q}$, as reported for instance in [8]. According to the authors' experience, it is the cavitation coefficient that mostly determines the choice of the specific speed in hydraulic turbines, in order to avoid excessive submergence of the unit, necessary to prevent cavitation, in relation to the head. In case of very low heads, and compact horizontal axis propeller axial turbines, which operate, submerged, it is not only the cavitation coefficient that determines the limitation to specific speed but also the loss of kinetic energy at the exit that even if reduced by the adoption of a diffuser of important dimensions, still remains a significant percentage of the low available net head. Because of the above considerations, for the design of the new compact propeller turbine of the present application, considering a design net head of $4 \mathrm{~m}$ and a possible variation range for net head applicability from $2 \mathrm{~m}$ to $8 \mathrm{~m}$ (obtainable by rotational speed variation), the choice of specific speed is limited to a value in the range from 200 to 220 and scalable dimensions with the propeller diameter in the range $D_{1 T I P}$ from $800 \mathrm{~mm}$ to $1500 \mathrm{~mm}$. The fundamental design parameters for turbomachinery design are the head or work coefficient $\psi=\frac{2 g H_{n}}{u_{1 T I P}^{2}}$ and the flow coefficient $\varphi=\frac{c_{x}}{u_{1 T I P}}$, which can be related to the specific speed $n_{q}$ through empirical correlations based on previous successful realization. Considering the specific speed variation previously defined, according to authors' experience, $\psi$ varies between 0.4 and 0.3 and $\varphi$ between 0.28 and 0.36 . Once that one of the two fundamental parameter is chosen, the other is automatically determined. In designing low head turbines, once that the head is fixed, the first design objective is to increase, as much as possible, the flow rate $Q$ that the turbine can deliver, in order to increase the useful output 
power. If the specific speed $n_{q}$ is already fixed, it means that the increase of $Q$ is compatible only with a decrease of the rotational speed, which means an increase of the blade aerodynamic loading and consequently of the profile losses and an higher value of $c_{x}$, which in turn can results in an increase of the kinetic energy losses at the exit. While the aerodynamic losses, that are in charge of the turbine, affect the total-to-total efficiency $\eta_{t-t}$, the discharge kinetic energy losses are in charge to the plant. However, both reduce the useful output power, which is the final target of the project:

$$
P_{u}=\rho g H_{n} Q \eta_{t-t} \eta_{m . e .}\left(1-\frac{H_{k e l}}{H_{n}}\right)
$$

In order to keep the head reduction coefficient $\left(1-H_{k e l} / H_{n}\right)$ to an acceptable value, unless the exceeding low design net head, a quite long exit conical diffuser is foreseen with a relative length $L / D_{1 T I P}$ of at least 5 and an area ratio of about 3 [9]. Given a global value of electric and mechanical efficiency $\eta_{\text {m.e. }}$ of 0.95 , for the above given design criteria and choices, reasonable target efficiency values can be $\eta_{t-t}=0.90$ and $\left(1-H_{k e l} / H_{n}\right)=0.94$, which need to be verified after design. The other design criteria are the runner hub to tip ratio in the range $0.44-0.40$ for high specific speed propellers, the axial velocity ratio of 2.0 from the turbine intake to the runner blade inlet section and an estimated bulb/blade tip diameter ratio of 0.8 . The runner blades will be manufactured by casting and machining and can be, therefore, designed twisted, according to the radial equilibrium equations NISRE. The vanes of the distributor, instead, should be cylindrical, in order to be simply manufactured by sheet metal calendering in order to obtain the correct spanwise exit flow angle distribution and then welded to hub and shroud surfaces [10]. For the rotor, a four-blade configuration has been chosen in order to limit the rotor blade axial chord and to keep a moderate profile winding at the hub. Pitch to chord ratio spanwise distribution has been determined utilizing the Zweifel criterion for the aerodynamic loading [11], verifying that the suction side pressure coefficient distribution, for each profile, would be suitable for moderate profile boundary layer development and would verify the value of the targeted design Thoma cavitation coefficient [12], to allow a cavitation free operation at the maximum rated head assumed as acceptable for this turbine series $\left(H_{n}=8.0 \mathrm{~m}\right)$. Span-wise distributions of the exit blade and vane flow angle deviations, necessary for the determination of the trailing edge blade and vane metal angle, have been evaluated iteratively from 3D CFD analysis software Numeca [13], as explained in the following paragraph.

\section{Turbine design}

From the design criteria defined in the previous paragraph, a first guess meridional sketch of the turbine can be obtained. In Fig. 1, the meridional channel of the machine is reported, with the characteristic sections to define the stator and rotor blades parameters.

Table 1. Propeller turbine main geometrical characteristic parameters.

\begin{tabular}{|c|c|}
\hline $\mathrm{D}_{\text {IN }} / \mathrm{D}_{1 \mathrm{TIP}}$ & 1.5 \\
\hline $\mathrm{D}_{1 \mathrm{HUB}} / \mathrm{D}_{1 \mathrm{TIP}}$ & 0.4 \\
\hline $\mathrm{D}_{1^{\prime} \mathrm{HUB}} / \mathrm{D}_{1 \mathrm{TIP}}$ & 0.7 \\
\hline $\mathrm{D}_{1^{\prime} \mathrm{TIP}} / \mathrm{D}_{1 \mathrm{TIP}}$ & 1.2 \\
\hline
\end{tabular}




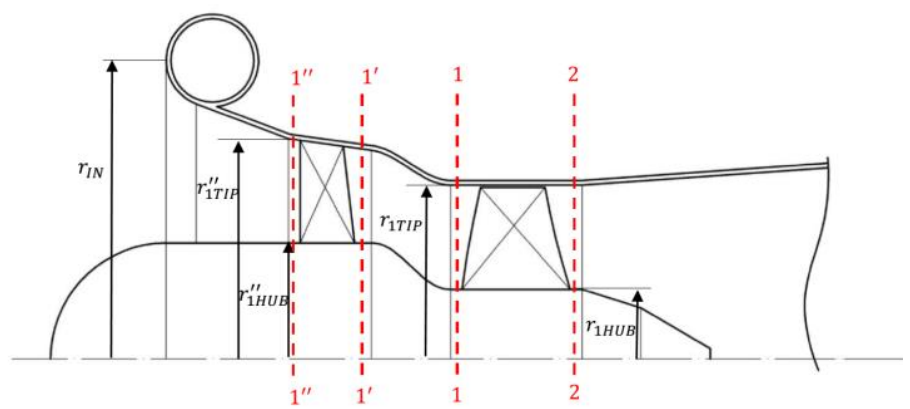

Fig. 1. Meridional channel of the machine and characteristic sections.

\subsection{Flow angles calculation}

For starting the turbine design, it is assumed that the net head $H_{n}$ and the reference diameter $D_{1 T I P}$ are known and the flow coefficient $\varphi$ and the head coefficient $\psi$ are fixed. The work coefficient can also be expressed as a function of the work exchange, that is, after having introduced the total to total turbine efficiency, $\eta_{t-t}$ :

$$
\psi=\frac{2 g H}{u_{1 T I P}^{2}}=\frac{2 W}{\eta_{t-t} u_{1 T I P}^{2}}=\frac{2 u_{1}\left(c_{\vartheta 1}-c_{\vartheta 2}\right)}{\eta_{t-t} u_{1 T I P}^{2}}
$$

From this expression, it is possible to derive the expression of the tangential component of absolute flow velocity at the inlet, in function of the radius, in case of null absolute tangential velocity component at the exit. For the hypothesis of constant work along the spanwise direction of the blade, e.g. free vortex design, the axial velocity component is constant along the span and can be easily evaluated from the $\varphi$ coefficient. It is therefore possible to evaluate the absolute and relative flow angles at rotor inlet section. The relative flow angles at rotor outlet are directly determined by the maximum efficiency condition, which sets $\alpha_{2}=0$

\subsection{Vane and blade metallic angle evaluation}

Once the absolute flow angles have been identified, it is necessary to evaluate the vane metallic angles suitable to realize the desired deflection. For both stator and rotor blade case, the flow deviation angle is evaluated by means of two-dimensional blade to blade calculations. In particular, the flow in the three characteristic blade sections at hub, mid-span and tip is simulated with a CFD solver [13]. For stator row, circular arc shaped blades have been employed. This shape is suitable in the current case, where the need to make simple machines, although with high efficiency, requires the use of blades with easy-to-manufacture and thus low cost geometry. The metallic angles at guide vane outlet $\left(\alpha_{c 1}\right)$ can be evaluated by adding the CFD obtained flow angle deviation to the stator outlet absolute flow angle.

By employing a constant blade thickness $(\mathrm{t})$, determined by the structural stress analysis, an elliptical leading edge and a tapered and rounded trailing edge, the stator blade shape, stacked on the leading edge, is completely defined (Fig. 2). 

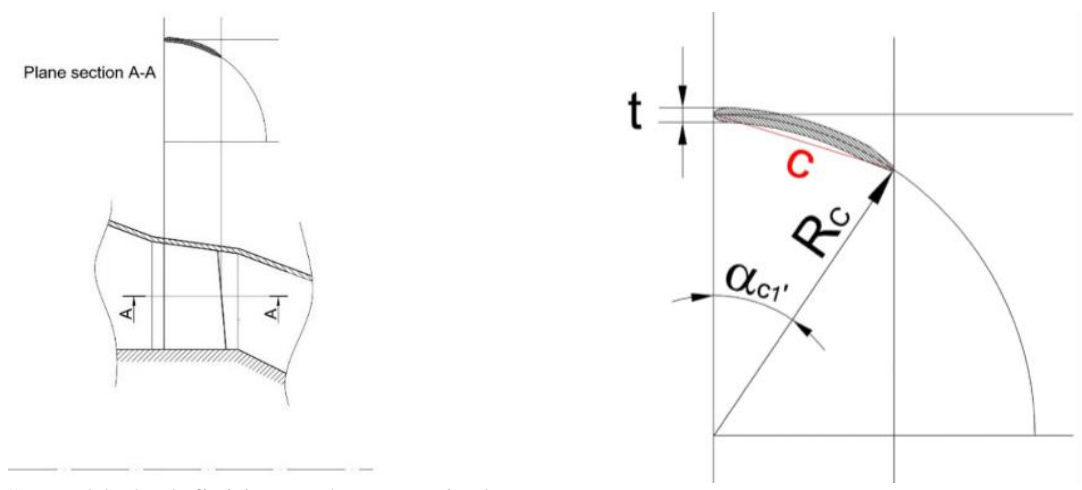

Fig. 2. Stator blade definition and geometrical parameters.

Also for the rotor blades, the flow deviation angle is evaluated by means of twodimensional blade to blade calculations. Once obtained the real flow angle, the blade geometry is modified in order to compensate the flow deviation angle. The procedure is repeated for a few iterations in order to obtain the desired flow angle. Once obtained the blade metallic angles for both stator and rotor blades, it is necessary to define the profile camber line curvature law and profile maximum thickness and thickness distribution from hub to tip. These values are assigned from previous successful realizations, after structural stress and vibrational analysis verification. Finally, to obtain the vane and blade final geometries it is necessary to define the profile stacking laws. In particular, as previously mentioned, the vanes of the distributor are cylindrical, and, for simplicity, the blade to blade sections are stacked on the leading edge. The runner blades sections, instead, are stacked on the centre of gravity. The 3D model obtained through the previously developed design procedure is depicted in Fig. 3.
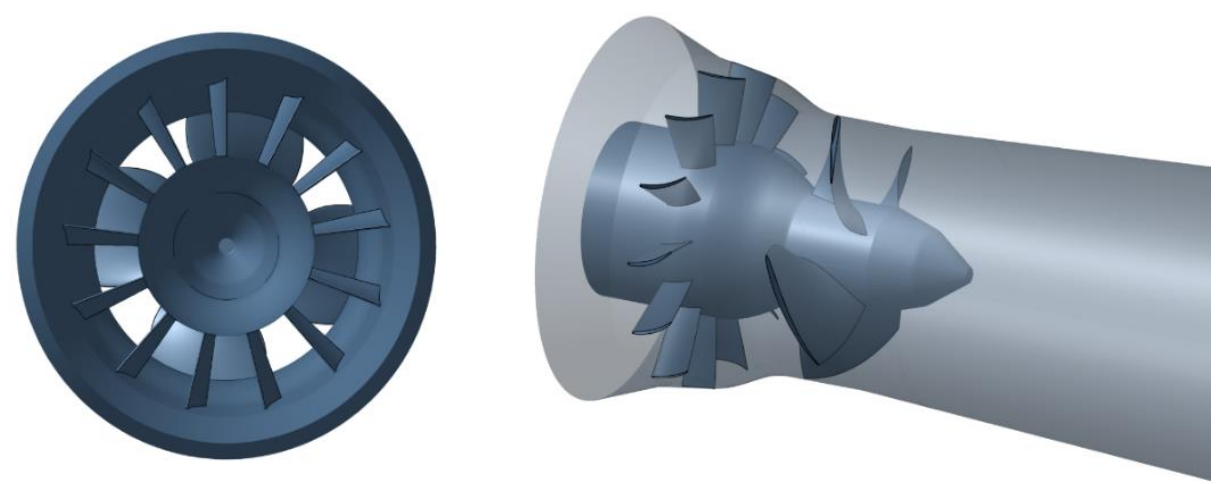

Fig. 3. 3D model of the propeller turbine: frontal and axonometric view.

\section{Numerical simulation and results}

The turbine model obtained by preliminary design coupled with the CFD iterative angles evaluation procedure has been analysed with a 3D complete RANS solver. The discretization in space is based on a cell centred control volume approach, while a Multi stage Runge-Kutta scheme with local time stepping is adopted for time discretization, associated with multigrid technique [13]. The computational mesh (

Fig. 4) was prepared in Autogrid5 and consists of about 4.7 million nodes. The modeller employs a multi block approach with $\mathrm{O}-4-\mathrm{H}$ mesh topology for the stator and rotor rows and 
extends to the simulation of the diffuser tube flow, by means of a suitable mesh capable of managing the condition of singularity on the axis. The mixing plane approach has been adopted to manage the passage of information from the stator frame to the rotor frame.

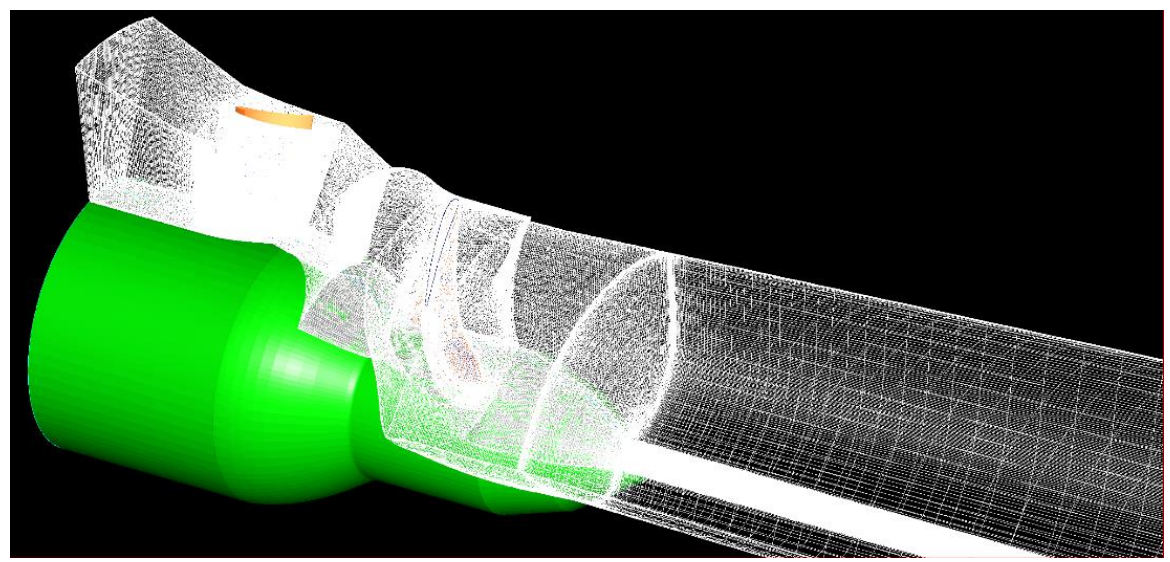

Fig. 4 Propeller turbine mesh.

For turbulence modelling, the simple one equation Spalart-Allmaras model was employed in order to obtain a simple yet reliable turbulence effects evaluation $[14,15]$. The height of the first cell near the solid walls was chosen, for each row, so that the value of the non-dimensional wall coordinate $y^{+}$was around one. As boundary conditions, at inlet section the mass flow rate, the static temperature and flow direction is set, while at outlet section the averaged static pressure, caused by the hydrostatic head at the outlet, is specified. The inlet velocity direction, in particular, is considered without swirl, while meridional angle is assigned with a linearly interpolating law that impose null value at hub and inlet duct slope at shroud. Since the solver employs a density based approach, the Hakimi preconditioning model was employed. The calculation takes about 4 hours to reach convergence. The final geometry is obtained after few iterations with limited changes of the vane, blade and hub geometries. These modifications are carried out by evaluating the spanwise distribution of flow angles downstream stator and rotor blades with the objective to obtain the flow angle distributions, defined by NISRE design procedure, which assure the target work exchange and flow rate. Table 2 shows the final 3D CFD results concerning the overall performance of the final turbine geometry, obtained as result from iterative procedure.

Table 2. Propeller turbine main operating parameters.

\begin{tabular}{|c|c|c|}
\hline & Final 3D simulation & Design targets \\
\hline$n_{q}$ & 211 & 212 \\
\hline$\varphi[-]$ & 0.290 & 0.290 \\
\hline$\psi[-]$ & 0.320 & 0.318 \\
\hline$\eta_{t-t}[-]$ & 0.907 & 0.900 \\
\hline$\left(1-H_{k e l} / H_{n}\right)[-]$ & 0.942 & 0.940 \\
\hline
\end{tabular}

The machine is able to achieve the target net head using the target flow rate calculated by preliminary design, as can be seen from the really close values of flow and work coefficient. The numerical simulation provides a total to total efficiency of 0.907 , calculated as: 


$$
\eta_{t-t}=\frac{\rho \omega T}{\Delta p_{t} \dot{m}}
$$

This value is slightly higher than the preliminary target value of 0.90 , which indicates a precautionary design procedure. Similarly, the head reduction coefficient, due to kinetic energy exit loss, predicted from the CFD simulation, is slightly better than that of the design target, within the range of the attended results. In

Fig. 5, the span wise distributions of the flow angles, normalized with respect to their maximum value, obtained by the CFD calculation (lines), are reported in correspondence of the sections of interest and compared with the values of the flow angles set as target from the design NISRE equation (points).

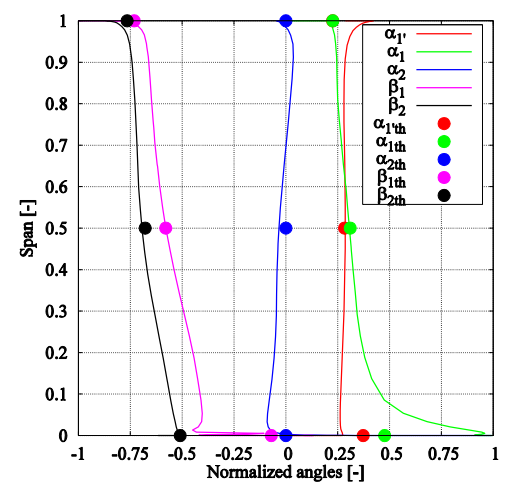

Fig. 5. Spanwise distributions of CFD calculated and theoretical design flow angles.

As it can be seen, the agreement between the target values and those obtained from the CFD simulation (employing the flow angle deviation corrections) is satisfactory. In Fig. 6 the non-dimensional axial and tangential velocity components upstream and downstream the runner blade (i.e. in sections 1 and 2) are presented.

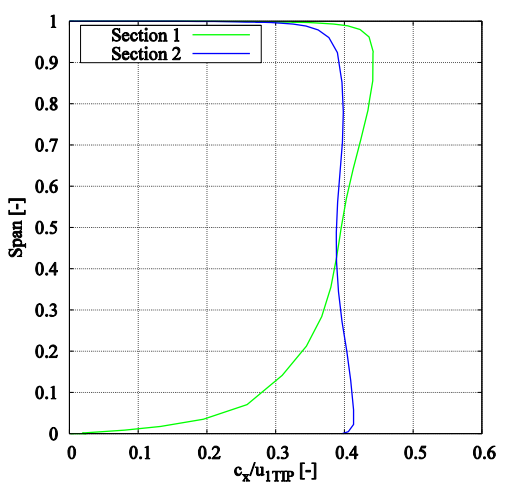

(a)

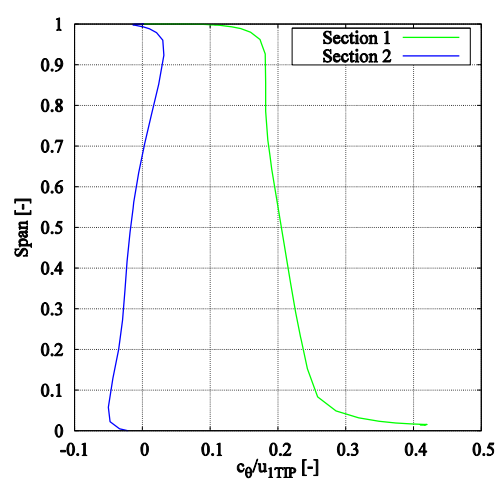

(b)

Fig. 6. Spanwise distributions of non-dimensional axial (a) and tangential (b) velocity components upstream and downstream the runner blade.

The distributions of axial and tangential components allow to better evaluate the mass flow rate and the work exchange distributions along the spanwise direction and to verify the reliability of preliminary design method. Finally, as example of CFD local results, 
Fig. 7 shows the contour fields for hub, midspan and tip sections of the rotor blade of the relative total pressure coefficient, representing the rotor losses, defined as:

$$
\omega_{p}=\frac{p_{t r 1}-p_{t r}}{p_{d 2}}
$$

Hub

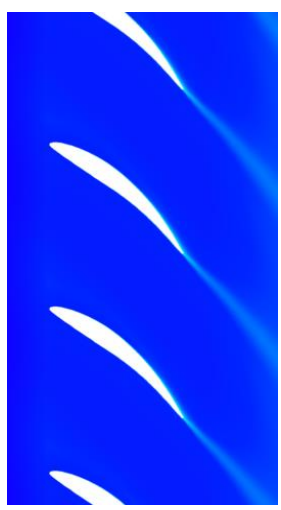

Midspan

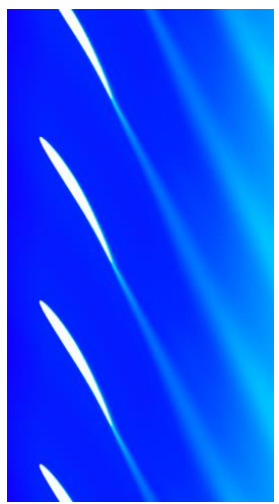

Tip

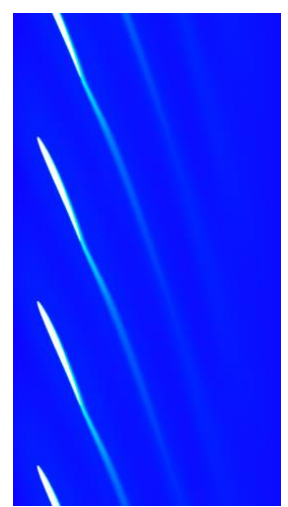

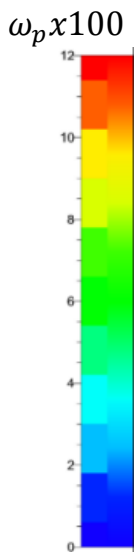

Fig. 7. Blade to blade relative total pressure coefficient colour plot for runner blade to blade hub, mid span and tip sections.

As can be noted, the losses generated by the boundary layer developments is rather limited, indicating a reasonable inlet incidence and exit flow deviation and adequate choice of the blade pitch to chord ratio in relation to the design flow deflection.

\section{Conclusions}

A preliminary design procedure for a compact, simplified, high specific speed hydraulic propeller turbine has been proposed and developed. The procedure, based on the use of the classical relationships and of the NISRE equation for axial turbines, supported and integrated by the use of dedicated CFD simulations for the evaluation of the deviation effect of the flow angles, was capable to define the geometry of the turbine. The geometry thus obtained, was used to generate the 3D model necessary to perform the CFD simulation of the entire turbine. The results of the CFD detailed analysis have been used iteratively to refine the preliminary design and effectively modify the geometry of the vane, blade profiles and hub geometry. This procedure made possible to accomplish, finally, the design target requirements including the turbine hydraulic efficiency and non-dimensional design operating parameters.

\section{References}

1. REN21. Renewables 2018 Global Status Report, ISBN 978-3-9818911-3-3M (2018)

2. http://voith.com/corp-en/hydropower-components/streamdiver.html

3. https://www.andritz.com/hy-hydromatrix

4. C. Vu, T. Koller, M. Gauthier, M. Deschênes, Flow simulation and efficiency hill chart prediction for a Propeller turbine (IOP Conference Series: Earth and Environmental Science, 2010) 
5. M. Morgut, D. Jošt, A. Škerlavaj, E. Nobile, G. Contento, Journal of Mechanical Engineering 64, 9, 543-554 (2018)

6. W. C. Schleicher, J. D. Riglin A. Oztekin, Renewable Energy 76, 234-241 (2015)

7. D. Thoma, Bereich Zeitschrift des Vereines deutscher Ingenieure 81, 972 (1937)

8. F. de Siervo, F. de Leva, Modern Trends in Selecting and Designing Kaplan Turbines (Water Power and Dam Construction, 1977)

9. G. Sovran, E. D. Klomp, Experimentally Determined Optimum Geometries for Rectilinear Diffusers with Rectangular Conical or Annular Cross-Section (Fluid Mechanics of Internal Flow, G. Sovran ed., 270-319, 1967)

10. A. Satta, M. Ubaldi, P. Zunino, Design of Axial Turbines for Mini-Hydro Plants, ASME, (Fourth International Symposium on Hydro Power Fluid Machinery-FED, 43, 29-36, 1989)

11. E. Dick, Fundamentals of Turbomachines (2015)

12. O. Ceravola, M. Ubaldi, P. Zunino, A. Satta, La Houille Blanche 7/8, 579-587 (1982)

13. NUMECA, User manuals. Academic R\&D license (2019)

14. Sh. Derakhshan, A. Mostafavi, Optimization of GAMM Francis Turbine Runner (Version 4986, 2011)

15. D. Borkowski, M. Węgiel, P. Ocłoñ, T. Węgiel, Web of Conf. 240, 05002 (2018) 